\title{
Current responsivity of semiconductor superlattice THz-photon detectors
}

\author{
Ignatov, Anatoly A.; Jauho, Antti-Pekka
}

Published in:

Journal of Applied Physics

Link to article, DOI:

$10.1063 / 1.369728$

Publication date:

1999

Document Version

Publisher's PDF, also known as Version of record

Link back to DTU Orbit

Citation (APA):

Ignatov, A. A., \& Jauho, A-P. (1999). Current responsivity of semiconductor superlattice THz-photon detectors. Journal of Applied Physics, 85(7), 3643-3654. https://doi.org/10.1063/1.369728

\section{General rights}

Copyright and moral rights for the publications made accessible in the public portal are retained by the authors and/or other copyright owners and it is a condition of accessing publications that users recognise and abide by the legal requirements associated with these rights.

- Users may download and print one copy of any publication from the public portal for the purpose of private study or research.

- You may not further distribute the material or use it for any profit-making activity or commercial gain

- You may freely distribute the URL identifying the publication in the public portal

If you believe that this document breaches copyright please contact us providing details, and we will remove access to the work immediately and investigate your claim 


\title{
Current responsivity of semiconductor superlattice THz-photon detectors
}

\author{
Anatoly A. Ignatov a) and Antti-Pekka Jauho \\ Mikroelektronik Centret, Technical University of Denmark, Building 345 East, DK-2800 Lyngby, Denmark
}

(Received 10 September 1998; accepted for publication 4 January 1999)

\begin{abstract}
The current responsivity of a semiconductor superlattice THz-photon detector is calculated using an equivalent circuit model which takes into account the finite matching efficiency between a detector antenna and the superlattice in the presence of parasitic losses. Calculations performed for currently available superlattice diodes show that both the magnitudes and the roll-off frequencies of the responsivity are strongly influenced by an excitation of hybrid plasma-Bloch oscillations which are found to be eigenmodes of the system in the THz-frequency band. The expected room temperature values of the responsivity (2-3 A/W in the $1-3 \mathrm{THz}$-frequency band) range up to several percents of the quantum efficiency $e / \hbar \omega$ of an ideal superconductor tunnel junction detector. Properly designed semiconductor superlattice detectors may thus demonstrate better room temperature THz-photon responsivity than conventional Schottky junction devices. (C) 1999 American Institute of Physics. [S0021-8979(99)03807-4]
\end{abstract}

\section{INTRODUCTION}

Theoretical investigations of high-frequency properties of semiconductor superlattices have been carried out for almost thirty years starting from the trail-blazing works by Esaki and Tsu. ${ }^{1,2}$ They proposed to prepare superlattices made of different semiconductor materials in order to realize an artificial periodical system that would allow to observe Bloch oscillations due to Bragg reflection of electrons from the boundaries of the Brillouin zone. Making use of the semiclassical Boltzmann equation they found that the dc current-voltage characteristics of the superlattice should show a negative differential conductance in sufficiently strong electric fields, namely, when the Bloch frequency $\Omega_{B}=e E d / \hbar$ (where $e$ is the electron's charge, $E$ is the dc electric field in the superlattice, $d$ is the superlattice period, $\hbar$ is the Planck's constant) becomes comparable to the effective scattering frequency $\nu .{ }^{1}$ They propose to make use of the negative differential conductance for development of an oscillator in the THz-frequency band ${ }^{1}$ and also suggested that the superlattice might possess promising properties as an artificial material for nonlinear mixing of photons. ${ }^{2}$

Later, several papers have proposed ${ }^{3-5}$ to probe Bloch oscillations in a superlattice in a strong dc electric field by applying an additional strong ac field. Self-induced transparency, ${ }^{3} \mathrm{dc}$ current suppression by a strong ac field causing dynamical localization of carriers, ${ }^{3-16}$ absolute negative conductance, ${ }^{3}$ and current harmonics generation with oscillating power dependence $e^{3,5}$ have been predicted to occur in the presence of resonant interaction between Bloch oscillating electrons and a strong THz-frequency radiation.

A profound analogy between the dynamics of electron wave packets in periodic systems and the dynamics of superconducting Josephson junctions ${ }^{17,18}$ was put forward to explain the main features of the superlattice $\mathrm{THz}$-frequency

\footnotetext{
a) Permanent address: Institute for Physics of Microstructures, Russian Acad-
} emy of Science, 603600 Nizhny Novgorod, Russia. response. ${ }^{18}$ It has been suggested, in particular, that the resonant steps on the dc current-voltage characteristics of the irradiated superlattices ${ }^{18}$ should occur in analogy with "Shapiro steps" normally observable in irradiated superconducting junctions. ${ }^{19}$ Several recent papers show that the curious behavior of electrons in the superlattices may give rise to a rich variety of new nonlinear phenomena occurring in the THz-field irradiated superlattices. ${ }^{20-25}$

In a number of papers Esaki-Tsu negative differential conductance was found to be experimentally realizable ${ }^{26,27}$ and has been carefully examined ${ }^{28-30}$ in the context of development of new millimeter-wave band $(0.03-0.3 \mathrm{THz})$ oscillators. On the other hand, the recent observations of a strong dc current suppression indicating dynamical localization of electrons, ${ }^{31}$ absolute negative conductance, ${ }^{32,33}$ and Shapiro steps on the dc current-voltage curve of the THzfield irradiated superlattices ${ }^{34}$ open the prospects for applications of the superlattices as novel solid state detectors operating in 1-10 THz-frequency band which the Bloch frequency in the superlattices normally belongs to. ${ }^{35}$

It has recently been estimated ${ }^{36}$ that the room temperature current responsivity of a superlattice detector ideally coupled to the $\mathrm{THz}$ photons can nearly reach the quantum efficiency $e / \hbar \omega$ (where $\omega$ is the incident radiation frequency) in the limit of high frequencies $\omega \gg \nu$. This value of the responsivity is being normally considered as a quantum limit for detectors based on superconducting tunnel junctions operating at low temperatures. ${ }^{19}$ For high frequencies the mechanism of the THz-photons detection in superlattices was described ${ }^{36}$ as a bulk superlattice effect caused by dynamical localization of electrons.

In this article we develop a self-consistent theory of the superlattice current responsivity. We apply the Boltzmann equation approach for describing the electron motion in the superlattice miniband ${ }^{3}$ and assume an equivalent circuit for the superlattice coupled to a broadband antenna (see Fig. 1), which is similar to the equivalent circuit used in resonant tunneling $^{37}$ and Schottky diode ${ }^{38}$ simulations. The suggested 


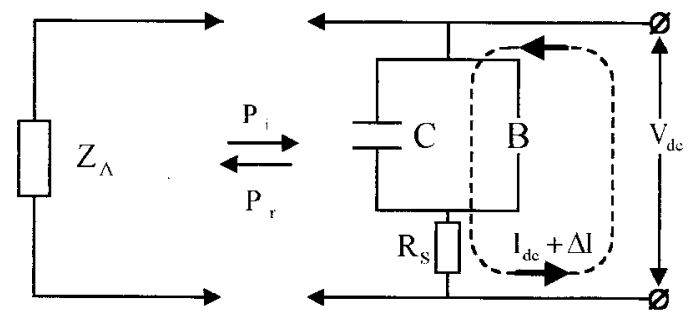

(b)

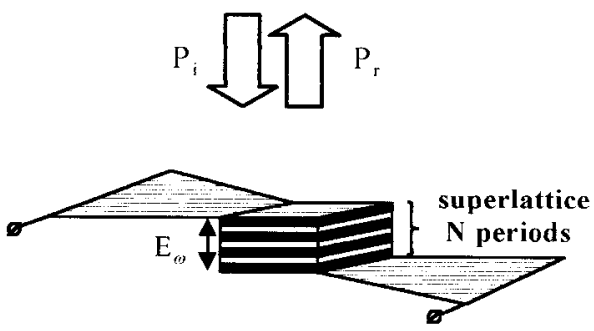

(a)

FIG. 1. (a) THz radiation coupled to an $n$-period semiconductor superlattice by a coplanar broadband bowtie antenna, $P_{i}$ and $P_{r}$ are the incident and reflected powers, respectively. (b) Equivalent circuit for a THz-photon detector with a dc voltage bias source: $B$-miniband electrons capable to perform Bloch oscillations, $C$-superlattice capacitance, $R_{S}$-parasitic series resistance, $Z_{A}$-bowtie antenna impedance, $V_{\mathrm{dc}}-\mathrm{dc}$ bias voltage.

equivalent circuit of the device allows one to treat microscopically the high-frequency response of the miniband electrons and, simultaneously, take into account a finite matching efficiency between the detector antenna and the superlattice in the presence of parasitic losses. Our analytic results lead to the identification of an important physical concept: the excitation of hybrid plasma-Bloch ${ }^{39}$ oscillations in the region of positive differential conductance of the superlattice. The numerical computations, performed for room temperature behavior of currently available superlattice diodes, show that both the magnitudes and the rolloff frequencies of the responsivity are strongly influenced by this effect. The excitation of the plasma-Bloch oscillations gives rise to a resonantlike dependence of the responsivity on the incident radiation frequency, improving essentially the coupling of the superlattice to the detector antenna. We will also show that peak current densities in the device and its geometrical dimensions should be properly optimized in order to get maximum responsivity for each frequency of the incident photons. Finally, we will present numerical estimates of the responsivity for the 1-4 THz frequency band and compare its value with the quantum efficiency $e / \hbar \omega$ of an ideal detector.

\section{DESCRIPTION OF THE MODEL}

For the description of the ac electron transport in a superlattice we use a quasiclassical wave packet treatment of the electron motion in a superlattice. ${ }^{1-3}$ The energy spectrum of electrons in a miniband is taken in a tight-binding approximation:

$$
\epsilon(\mathbf{p})=\frac{\Delta}{2}\left[1-\cos \left(\frac{p_{z} d}{\hbar}\right)\right]+\frac{p_{x}^{2}+p_{y}^{2}}{2 m},
$$

where $\Delta$ is the superlattice miniband width, $d$ is the superlattice period, $p_{z}$ is the quasimomentum of an electron along the superlattice axis (perpendicular to the layers), $p_{x}, p_{y}$ are the quasimomentum components along the superlattice layers, and $m$ is the effective mass of electrons along the superlattice layers.

The quasiclassical velocity $v_{z}\left(p_{z}\right)$ of an electron moving along the superlattice axis and the time derivative of the quasimomentum are given by the expressions

$$
\begin{aligned}
& v_{z}\left(p_{z}\right)=\frac{\partial \boldsymbol{\epsilon}(\mathbf{p})}{\partial p_{z}}=v_{0} \sin \left(\frac{p_{z} d}{\hbar}\right), \\
& \dot{p}_{z}=e E_{z}(t),
\end{aligned}
$$

where $e$ is the electron charge, $v_{0}=\Delta d / 2 \hbar$ is the maximum velocity of electrons along the superlattice axis, and $E_{z}(t)$ is the time-periodic electric field directed along the superlattice axis.

The electric current density $j_{z}(t)$ is calculated from the distribution function

$$
j_{z}(t)=e \int v_{z}\left(p_{z}\right) f(\mathbf{p}, t) \frac{2 d \mathbf{p}}{(2 \pi \hbar)^{3}},
$$

which satisfies the time-dependent Boltzmann equation

$$
\frac{\partial f(\mathbf{p}, t)}{\partial t}+e E_{z}(t) \frac{\partial f(\mathbf{p}, t)}{\partial p_{z}}=\left(\frac{\partial f}{\partial t}\right)_{\text {coll }} .
$$

Below we use the relaxation-time approximation for the collision integral ${ }^{1-3}$

$$
\left(\frac{\partial f}{\partial t}\right)_{\text {coll }}=-\frac{f(\mathbf{p}, t)-f_{0}(p)}{\tau},
$$

where $\tau$ is the constant relaxation time for electron's scattering, and $f_{0}(p)$ is the equilibrium distribution function.

The relaxation-time approximation is, of course, an oversimplification of the numerous scattering processes taking place in a real superlattice. Nevertheless, several papers ${ }^{40}$ have demonstrated that the phenomenon of dynamical localization can be described very well within this approximation when compared to the corresponding results obtained from a full-scale Monte Carlo simulation. This circumstance lends support to the simplified model for the collision integral. The great advantage of Eqs. (5) and (6) is that they allow an analytical calculation of the time-dependent current, to be used in the equivalent circuit analysis, and thus we can study in detail various parametric dependencies of the calculated quantities.

We would also like to emphasize here that the wave packet description of electron motion in a superlattice is valid if the following inequalities are fulfilled: $:^{3,41}$

$$
\begin{aligned}
& e E d \ll \Delta, \Delta_{G}, \\
& \hbar \omega \ll \Delta, \Delta_{G}, \\
& \hbar \nu \ll \Delta, \Delta_{G},
\end{aligned}
$$

where $\Delta_{G}$ is the width of the superlattice minigap, $\nu=1 / \tau$ is the electron's collision frequency, and $\omega$ is the characteristic frequency of the external ac field. We also assume that the 
electronic mean free path $\ell_{\mathrm{FP}}=v_{0} \tau=\Delta d / 2 \hbar \nu$ is smaller than the superlattice length $L$ in order to neglect the influence of the boundaries on the superlattice high-frequency properties.

\section{THEORETICAL FORMALISM}

\section{A. Path integral}

The exact solution of Eqs. (5) and (6) for arbitrary timedependent electric field can be presented in the form of a path integral: ${ }^{42}$

$$
\begin{aligned}
f(\mathbf{p}, t)= & \int_{-\infty}^{t} \nu d t_{1} \exp \left[-\nu\left(t-t_{1}\right)\right] \\
& \times f_{0}\left(p_{z}-\int_{t_{1}}^{t} e E_{z}\left(t_{2}\right) d t_{2}\right) .
\end{aligned}
$$

Using Eqs. (4) and (10) we find the time-dependent current $I(t)$ describing ac transport in a superlattice (SL) with electron performing ballistic motion in a miniband according to the acceleration theorem and suffering scattering: ${ }^{2,3}$

$$
\begin{aligned}
I(t)= & 2 I_{p} \int_{-\infty}^{t} \nu d t_{1} \exp \left[-\nu\left(t-t_{1}\right)\right] \\
& \times \sin \left(\frac{e}{N \hbar} \int_{t_{1}}^{t} V\left(t_{2}\right) d t_{2}\right),
\end{aligned}
$$

where $V(t)=L E_{z}(t)$ is the voltage across the superlattice perpendicular to the layers, $L=N d$ is the superlattice length, $N$ is the number of periods in the superlattice sample, $I_{p}$ $=S j_{p}, S=\pi a^{2}$ is the area of the superlattice, $a$ is the superlattice mesa radius, and

$$
j_{p}=e \frac{v_{0}}{2} \int \frac{2 d \mathbf{p}}{(2 \pi \hbar)^{3}} \cos \left(\frac{p_{z} d}{\hbar}\right) f_{0}(p),
$$

is the characteristic current density. The integration over $p_{z}$ in Eq. (12) must be carried out over the Brillouin zone $-\pi \hbar / d \leqslant p_{z} \leqslant \pi \hbar / d$.

In particular, for a de voltage $V_{\mathrm{SL}}$ applied to the superlattice Eq. (11) results in the Esaki-Tsu ${ }^{1}$ current-voltage curve

$$
I_{\mathrm{dc}}^{\mathrm{SL}}\left(V_{\mathrm{SL}}\right)=2 I_{P} \frac{\left(V_{\mathrm{SL}} / V_{P}\right)}{1+\left(V_{\mathrm{SL}} / V_{P}\right)^{2}} .
$$

At peak voltage $V_{\mathrm{SL}}=V_{P}=N \hbar \nu / e$ or, equivalently, at peak electric field $E_{\mathrm{SL}}=E_{P}=\hbar \nu / e d$, the current in the superlattice reaches its maximum (peak) value $I_{P}$, so that $j_{P}$ can be defined as the peak current density.

The peak current density $j_{P}$ and the scattering frequency $\nu$ can be considered as the main parameters of the employed model. They can readily be estimated from experimentally measured or numerically simulated values of $I_{P}$ and $V_{P}$. For both degenerate and nondegenerate electron gas one gets ${ }^{2,3}$

$$
j_{P}=e n \frac{v_{0}}{2},
$$

if $\Delta \gg k T, \epsilon_{F}$, where $k T$ is the equilibrium thermal excitation energy, $\epsilon_{F}=\hbar^{2}\left(3 \pi^{2} n\right)^{2 / 3} /\left(2 m_{\text {eff }}\right)$ is the Fermi energy of de- generate electrons, $n=\int f_{0}(\mathbf{p}) 2 d \mathbf{p} /(2 \pi \hbar)^{3}$ is the charge carrier density, $m_{\text {eff }}=m_{z z}^{1 / 3} m^{2 / 3}$ is the density of states effective mass near the miniband bottom, and $m_{z z}=2 \hbar^{2} / \Delta d^{2}$ is the effective mass of electrons along the superlattice axis. In the particular case of the Boltzmann equilibrium distribution function Eq. (12) yields ${ }^{3} j_{P}=\left(e n v_{0} / 2\right)\left[I_{1}(\Delta / 2 k T) /\right.$ $\left.I_{0}(\Delta / 2 k T)\right]$, where $I_{0,1}$ are the modified Bessel functions.

\section{B. Monochromatic excitation}

We now suppose that in addition to the dc voltage $V_{\mathrm{SL}}$, an alternating sinusoidal voltage with a complex amplitude $V_{\omega}$ is applied to the superlattice:

$$
V(t)=V_{\mathrm{SL}}+\frac{1}{2}\left[V_{\omega} \exp (i \omega t)+V_{\omega}^{*} \exp (-i \omega t)\right] .
$$

Generally, $V_{\mathrm{SL}}, V_{\omega}$ can be found from an analysis of the equivalent circuit given in Fig. 1. We write the ac voltage amplitude as $V_{\omega}=\left|V_{\omega}\right| e^{i \psi}$; both $\left|V_{\omega}\right|$ and $\psi$ can be obtained self-consistently taking account of reflection of the THz photons from the superlattice and their absorption in the series resistor $R_{S}$.

Making use Eq. (11) we obtain ${ }^{3}$

$$
I(t)=2 I_{P} \int_{0}^{\infty} \nu d t_{1} \exp \left(-\nu t_{1}\right) \sin \left[\frac{e V_{\mathrm{SL}}}{N \hbar} t_{1}+\Phi\left(t, t_{1}\right)\right]
$$

where

$$
\begin{aligned}
\Phi\left(t, t_{1}\right)= & \frac{e}{N \hbar \omega} \times \frac{1}{2}\left\{i V_{\omega} \exp (i \omega t)\right. \\
& \left.\times\left[\exp \left(-i \omega t_{1}\right)-1\right]+\text { c.c. }\right\} .
\end{aligned}
$$

According to Eq. (16), electrons in a superlattice miniband perform damped Bloch oscillations with the frequency $\Omega_{B}$ $=e V_{\mathrm{SL}} / N \hbar=e E_{\mathrm{SL}} d / \hbar$, and the phase $\Phi\left(t, t_{1}\right)$ modulated by the external ac voltage.

In the limit of $V_{\omega} \rightarrow 0$ Eq. (16) reduces to the dc currentvoltage curve given by Eq. (13). On the other hand, when the frequency of the ac voltage is small, $\omega \tau \ll 1$, we get

$$
\Phi\left(t, t_{1}\right)=\frac{e t_{1}}{N \hbar} \times \frac{1}{2}\left[V_{\omega} \exp (i \omega t)+\text { c.c. }\right],
$$

which corresponds to a slow modulation of the Bloch frequency by external voltage. In this case the current in the superlattice follows instantaneously the time-dependent ac voltage according to the dc current-voltage curve.

Equation (16) contains, as special cases the following results: (i) a harmonic voltage $V(t)\left(V_{\mathrm{SL}}=0\right)$ leads to dynamical localization, and current harmonics generation with oscillating power dependence; ${ }^{3}$ (ii) dc current-voltage characteristics of the irradiated superlattice $I_{\mathrm{dc}}\left(V_{\mathrm{SL}}, V_{\omega}\right)$ $=(\omega / 2 \pi) \int I(t) d t$ shows resonance features (Shapiro steps) leading to absolute negative conductance; $;^{3,5,7,18}$ (iii) and to generation of dc voltages (per one superlattice period) that are multiples of $\hbar \omega / e .^{13}$ 


\section{Method of perturbations}

Let us assume that the external ac voltage $V_{\omega}$ is so small that perturbation theory holds, while the dc voltage $V_{\mathrm{SL}}$ applied to the superlattice keeps its finite value. Expanding Eq. (16) around $V_{\mathrm{SL}}$ at $V_{\omega} \rightarrow 0$ in a Taylor series, we obtain the time-dependent electric current in the form:

$$
\begin{aligned}
I(t)= & I_{\mathrm{dc}}^{\mathrm{SL}}\left(V_{\mathrm{SL}}\right)+\frac{1}{2}\left[G_{\mathrm{ac}}^{\mathrm{SL}}\left(\omega, V_{\mathrm{SL}}\right) V_{\omega} e^{i \omega t}+\text { c.c. }\right] \\
& +\Delta I_{\mathrm{dc}}^{\mathrm{SL}}\left(\omega, V_{\mathrm{SL}}\right),
\end{aligned}
$$

where

$$
G_{\mathrm{ac}}^{\mathrm{SL}}\left(\omega, V_{\mathrm{SL}}\right)=G_{0} F_{1}\left(\omega, V_{\mathrm{SL}}\right),
$$

is the superlattice ac conductance, ${ }^{3} G_{0}=2 I_{P} / V_{P}$ is the superlattice conductance at $\omega \rightarrow 0, V_{\mathrm{SL}} \rightarrow 0$ :

$F_{1}\left(\omega, V_{\mathrm{SL}}\right)=\frac{1+i \omega \tau-\left(V_{\mathrm{SL}} / V_{P}\right)^{2}}{\left[1+\left(V_{\mathrm{SL}} / V_{P}\right)^{2}\right]\left[(1+i \omega \tau)^{2}+\left(V_{\mathrm{SL}} / V_{P}\right)^{2}\right]}$,

is a dimensionless function describing the dependence of the superlattice conductance both on frequency and applied dc voltage (field), and

$$
\Delta I_{\mathrm{DV}}^{\mathrm{SL}}\left(\omega, V_{\mathrm{SL}}\right)=\frac{1}{4}\left|V_{\omega}\right|^{2} F_{2}\left(\omega, V_{\mathrm{SL}}\right),
$$

where

$$
\begin{aligned}
F_{2}\left(\omega, V_{\mathrm{SL}}\right) & =\frac{I_{\mathrm{dc}}^{\mathrm{SL}}\left(V_{\mathrm{SL}}+N \hbar \omega / e\right)-2 I_{\mathrm{dc}}^{\mathrm{SL}}\left(V_{\mathrm{SL}}\right)+I_{\mathrm{dc}}^{\mathrm{SL}}\left(V_{\mathrm{SL}}-N \hbar \omega / e\right)}{(N \hbar \omega / e)^{2}}, \\
& =-\frac{4 I_{P}}{V_{P}^{2}} \frac{\left(V_{\mathrm{SL}} / V_{P}\right)\left[3+(\omega \tau)^{2}-\left(V_{\mathrm{SL}} / V_{P}\right)^{2}\right]}{\left[1+\left(V_{\mathrm{SL}} / V_{P}\right)^{2}\right]\left[1+\left(V_{\mathrm{SL}} / V_{P}+\omega \tau\right)^{2}\right]\left[1+\left(V_{\mathrm{SL}} / V_{P}-\omega \tau\right)^{2}\right]},
\end{aligned}
$$

is the change in the dc current in a superlattice caused by THz photons. ${ }^{3}$

At $\omega \rightarrow 0$ Eq. (20) yields the de differential conductance of the superlattice

$$
\begin{aligned}
G_{\mathrm{ac}}^{\mathrm{SL}}\left(\omega \rightarrow 0, V_{\mathrm{SL}}\right) & =d I_{\mathrm{dc}}^{\mathrm{SL}}\left(V_{\mathrm{SL}}\right) / d V_{\mathrm{SL}} \\
& =G_{0} \frac{1-\left(V_{\mathrm{SL}} / V_{P}\right)^{2}}{\left[1+\left(V_{\mathrm{SL}} / V_{P}\right)^{2}\right]^{2}},
\end{aligned}
$$

while at $V_{\mathrm{SL}} \rightarrow 0$ one gets the well-known Drude formula for the ac conductivity of the electron gas $\sigma(\omega)$

$$
\sigma(\omega)=\frac{\sigma_{0}}{1+i \omega \tau}
$$

where the small-field dc conductivity of the superlattice $\sigma_{0}$ can be given in terms of the dc conductance $\sigma_{0}=G_{0} L / S$ or, equivalently, in terms of peak current density and the peak electric field

$$
\sigma_{0}=2 \frac{j_{P}}{E_{P}}
$$

According to Eqs. (15) and (19) the ac power $P_{\text {abs }}^{\mathrm{SL}}$ absorbed in the superlattice is

$$
P_{\mathrm{abs}}^{\mathrm{SL}}=\frac{1}{2} \operatorname{Re} G_{\mathrm{ac}}^{\mathrm{SL}}\left(\omega, V_{\mathrm{SL}}\right)\left|V_{\omega}\right|^{2},
$$

where the real part of the superlattice conductance can be presented in analogy with Eq. (23) in a finite difference form $^{3,43}$

$$
\begin{aligned}
\operatorname{Re} G_{\mathrm{ac}}^{\mathrm{SL}}\left(\omega, V_{\mathrm{SL}}\right) \\
=\frac{e}{2 N \hbar \omega}\left[I_{\mathrm{dc}}^{\mathrm{SL}}\left(V_{\mathrm{SL}}+N \hbar \omega / e\right)-I_{\mathrm{dc}}^{\mathrm{SL}}\left(V_{\mathrm{SL}}-N \hbar \omega / e\right)\right] \\
=\frac{2 I_{P}}{V_{P}} \frac{1+(\omega \tau)^{2}-\left(V_{\mathrm{SL}} / V_{P}\right)^{2}}{\left[1+\left(V_{\mathrm{SL}} / V_{P}+\omega \tau\right)^{2}\right]\left[1+\left(V_{\mathrm{SL}} / V_{P}-\omega \tau\right)^{2}\right]}
\end{aligned}
$$

We note here that our calculations assume uniform dc/ac electric fields inside the superlattice. This assumption is justified for the subthreshold dc electric fields $V_{\mathrm{SL}} \leqslant V_{P}$, and/or for ac field frequencies larger than the transit-time frequency of space-charge waves $\omega \geqslant v_{0} / L$ in the superlattice. ${ }^{39}$

\section{Stimulated emission and positive photocurrent}

Equation (28) for the superlattice conductance demonstrates a possibility of resonant interaction of the $\mathrm{THz}$ photons with electrons performing damped Bloch oscillations in the superlattice. For $\omega \tau \gg 1$ the condition for the resonance has a form $\omega= \pm\left(V_{\mathrm{SL}} / V_{P}\right) / \tau$, which can be equivalently expressed as $\omega= \pm \Omega_{B}$. At dc electric fields $E_{\mathrm{SL}}$ $\geqslant E_{P} \sqrt{1+(\omega \tau)^{2}}$ the real part of the superlattice conductance starts to be negative which corresponds to negative absorption (stimulated emission) of the $\mathrm{THz}$ photons. In the limit $\omega \tau \gg 1$ photons with frequency $\omega>\Omega_{B}$ are absorbed and the ones having frequency $\omega<\Omega_{B}$ are emitted.

Equation (22) describes the dc current change in the superlattice under the influence of the THz photons. The standard result of the classical rectification theory ${ }^{19}$

$$
\Delta I_{\mathrm{dc}}^{\mathrm{SL}}\left(\omega \rightarrow 0, V_{\mathrm{SL}}\right)=\frac{1}{4}\left|V_{\omega}\right|^{2} I_{\mathrm{dc}}^{\mathrm{SL}}\left(V_{\mathrm{SL}}\right),
$$

where 


$$
I_{\mathrm{dc}}^{\mathrm{SL}}\left(V_{\mathrm{SL}}\right)=-4 \frac{I_{P}}{V_{P}^{2}} \frac{\left(V_{\mathrm{SL}} / V_{P}\right)\left[3-\left(V_{\mathrm{SL}} / V_{P}\right)^{2}\right]}{\left[1+\left(V_{\mathrm{SL}} / V_{P}\right)^{2}\right]^{3}},
$$

is the second derivative of the dc current-voltage curve of the superlattice, is obtained from Eq. (22) when the electron system relaxes during one period of the ac field.

At dc bias fields $E_{\mathrm{SL}}<E_{P} \sqrt{3+(\omega \tau)^{2}}$ the current change in the irradiated superlattice is negative which corresponds to the onset of suppression of the current due to dynamical localization of carriers. ${ }^{3-14,16}$ In the opposite case $E_{\mathrm{SL}}$ $>E_{P} \sqrt{3+(\omega \tau)^{2}}$ a positive current change (positive photocurrent) occurs. It is important to note that the condition of occurrence of the positive photocurrent implies, according to Eqs. (22) and (27), the existence of stimulated emission of photons from the superlattice, $P_{\mathrm{abs}}^{\mathrm{SL}}<0$.

The increase of the dc current in superlattices caused by stimulated emission of $\mathrm{THz}$ photons has recently been observed in experiments. ${ }^{34}$ These experimental results lend support to the validity of the theoretical formalism employed in this article for the analysis of the superlattice THz-field response.

\section{CURRENT RESPONSIVITY}

\section{A. Equivalent circuit calculation}

We define the current responsivity ${ }^{19}$ of the superlattice detector as the current change $\Delta I$ induced in the external dc circuit per incoming ac signal power $P_{i}$ :

$$
R_{i}\left(\omega, V_{\mathrm{SL}}\right)=\frac{\Delta I}{P_{i}} .
$$

This definition takes into account both the parasitic losses in the detector and the finite efficiency for impedance matching of the incoming signal into the superlattice diode. In what follows we use both the frequency $f=\omega / 2 \pi$ and the angular frequency $\omega$ notations.

According to Eqs. (22) and (27), in the small-signal approximation both the dc current change $\Delta I_{\mathrm{dc}}^{\mathrm{SL}}$ and the power $P_{\mathrm{abs}}^{\mathrm{SL}}$ absorbed in the superlattice are proportional to the square modulus of the complex voltage $\left|V_{\omega}\right|^{2}$. This circumstance permits us to calculate $\left|V_{\omega}\right|^{2}$ self-consistently for given values of the incoming power, making use a linear ac equivalent circuit analysis and, then, find the current responsivity $R_{i}\left(\omega, V_{\mathrm{SL}}\right)$.

The results of the calculation of the superlattice current responsivity $R_{i}\left(\omega, V_{\mathrm{SL}}\right)$ are presented in the following form:

$$
R_{i}\left(\omega, V_{\mathrm{SL}}\right)=\frac{R_{i}^{(0)}\left(\omega, V_{\mathrm{SL}}\right) A\left(\omega, V_{\mathrm{SL}}\right)}{1+R_{S}\left[d I_{\mathrm{dc}}^{\mathrm{SL}}\left(V_{\mathrm{SL}}\right) / d V_{\mathrm{SL}}\right]},
$$

where

$$
\begin{aligned}
& R_{i}^{(0)}\left(\omega, V_{\mathrm{SL}}\right) \\
& \quad=-\frac{e}{N \hbar \nu} \frac{\left(V_{\mathrm{SL}} / V_{P}\right)\left[3+(\omega \tau)^{2}-\left(V_{\mathrm{SL}} / V_{P}\right)^{2}\right]}{\left[1+\left(V_{\mathrm{SL}} / V_{P}\right)^{2}\right]\left[1+(\omega \tau)^{2}-\left(V_{\mathrm{SL}} / V_{P}\right)^{2}\right]},
\end{aligned}
$$

is the superlattice current responsivity under conditions of a perfect matching and neglecting parasitic losses, $R_{S} \rightarrow 0 .{ }^{36}$
The factor $A\left(\omega, V_{\mathrm{SL}}\right)$ in Eq. (32) describes the effect of the electrodynamical mismatch between the antenna and the superlattice and the signal absorption in the series resistance

$$
\begin{aligned}
A\left(\omega, V_{\mathrm{SL}}\right)= & {\left[1-\left|\frac{Z_{A}-\left[Z_{\mathrm{ac}}^{\mathrm{SL}}\left(\omega, V_{\mathrm{SL}}\right)+R_{S}\right]}{Z_{A}+\left[Z_{\mathrm{ac}}^{\mathrm{SL}}\left(\omega, V_{\mathrm{SL}}\right)+R_{S}\right]}\right|^{2}\right] } \\
& \times \frac{\operatorname{Re} Z_{\mathrm{ac}}^{\mathrm{SL}}\left(\omega, V_{S L}\right)}{\operatorname{Re} Z_{\mathrm{ac}}^{\mathrm{SL}}\left(\omega, V_{\mathrm{SL}}\right)+R_{S}} .
\end{aligned}
$$

The first factor in Eq. (34) describes the reflection of the THz photons due to mismatch of the antenna impedance $Z_{A}$ and the total impedance of the device $Z_{\mathrm{ac}}^{\mathrm{SL}}\left(\omega, V_{\mathrm{SL}}\right)+R_{S}$, with the second one being responsible for sharing of the absorbed power between the active part of the device described by the impedance $Z_{\mathrm{ac}}^{\mathrm{SL}}\left(\omega, V_{\mathrm{SL}}\right)$ and the series resistance $R_{S}$.

The superlattice impedance is defined as

$$
Z_{\mathrm{ac}}^{\mathrm{SL}}\left(\omega, V_{\mathrm{SL}}\right)=1 /\left[G_{\mathrm{ac}}^{\mathrm{SL}}\left(\omega, V_{\mathrm{SL}}\right)+i \omega C\right],
$$

where $G_{\mathrm{ac}}^{\mathrm{SL}}\left(\omega, V_{\mathrm{SL}}\right)$ is the superlattice conductance, $C$ $=\epsilon_{0} S / 4 \pi L$ is the capacitance of the superlattice, and $\epsilon_{0}$ is the average dielectric lattice constant.

Finally, the last factor in the denominator of Eq. (32) describes the redistribution of the external bias voltage $V_{\mathrm{dc}}$ between the dc differential resistance of the superlattice $\left[d I_{\mathrm{dc}}^{\mathrm{SL}}\left(V_{\mathrm{SL}}\right) / d V_{\mathrm{SL}}\right]^{-1}$ and the series resistance $R_{S}$, with the dc voltage drop on the superlattice $V_{\mathrm{SL}}$ being determined by the solution of the well-known load equation ${ }^{19}$

$$
V_{\mathrm{dc}}=V_{\mathrm{SL}}+I_{\mathrm{dc}}^{\mathrm{SL}}\left(V_{\mathrm{SL}}\right) R_{S} .
$$

\section{B. Classical rectification at high frequencies}

Suppose now that the following set of inequalities is satisfied

$$
\begin{aligned}
& \omega \tau \ll 1, \\
& R_{S} \ll R_{0}, \\
& \omega \gg 1 / \sqrt{R_{0} R_{S}} C,
\end{aligned}
$$

which implies that the frequency of the incident radiation $\omega$ is small in comparison with the scattering frequency of electrons $\nu$, series resistance $R_{S}$ is small in comparison with the small-field dc resistance of the superlattice $R_{0}=1 / G_{0}$, and the appropriate $R C$ time of the device is high enough. In this case Eq. (32) gives

$$
R_{i}\left(\omega, V_{\mathrm{SL}}\right)=2 I_{\mathrm{dc}}^{\mathrm{SL}}\left(V_{\mathrm{SL}}\right) \times \frac{Z_{A}}{\left(1+Z_{A} / R_{S}\right)^{2}} \times \frac{1}{\left(\omega R_{S} C\right)^{2}} .
$$

Equation (40) was obtained by Sollner $e t a l .{ }^{37}$ following Torrey's and Whitmer's approach ${ }^{38}$ developed for analysis of classical crystal rectifiers. They also took account of the mismatch between the antenna impedance $Z_{A}$ and the device impedance. It was applied to analyze detection of photons by resonant tunneling diodes in the THz-frequency range. This expression demonstrates that the responsivity of the classical rectifier at high frequencies is proportional to the second derivative of the dc current-voltage curve, and hence strongly decreases with increasing of frequency, $R_{i}\left(\omega, V_{\mathrm{SL}}\right) \propto \omega^{-2}$. 
The factor $1 / R_{S} C$ in Eq. (40) defines the rolloff frequency for the current responsivity and, consequently, capacitance of the device should be minimized in order to increase the responsivity in the high frequency range. The fast decrease of the responsivity with increasing of frequency can be attributed to imperfect matching of the device to the antenna impedance when incoming photons either are reflected from the device or are absorbed in the series resistor.

In our case Eq. (40) shows that the current responsivity of the superlattice can reach its maximum (negative) value at bias voltage $V_{\mathrm{SL}} \simeq 0.4 V_{P}$, i.e., in the region of the positive conductance. Furthermore, the responsivity of the superlattice does not depend on the length of the device because $V_{P} \propto L$ and $C \propto 1 / L$, and, finally, the responsivity is proportional to the current density $j_{P}$. Consequently, in order to increase the responsivity in the high-frequency range one should employ highly conductive superlattice samples for which inequalities [Eqs. (37)-(39)] may not be satisfied. In this case the interaction of the superlattice with the incoming radiation can be essentially changed by excitation of the eigenmodes in the superlattice device.

\section{Superlattice dielectric function: Hybridization of Bloch and plasma oscillations}

Let us analyze the condition of optimized matching of the superlattice to the incident radiation going back to the general Eq. (32). Assuming the limit of negligible series resistance $R_{S} \rightarrow 0$ this condition can be obtained from the solution of the equation

$$
Z_{\mathrm{ac}}^{\mathrm{SL}}\left(\omega, V_{\mathrm{SL}}\right)=Z_{A},
$$

for the complex frequency $\omega\left(V_{\mathrm{SL}}\right)$. This solution determines the resonant line position and the linewidth at which the absorption in the superlattice tends to its maximum value.

Using Eqs. (20) and (35) one can transform Eq. (41) to the following form:

$$
\epsilon\left(\omega, E_{\mathrm{SL}}\right)=\frac{\epsilon_{0}}{i \omega C Z_{A}},
$$

where

$$
\epsilon\left(\omega, E_{\mathrm{SL}}\right)=\epsilon_{0}+\frac{4 \pi \sigma_{0}}{i \omega} F_{1}\left(\omega, E_{\mathrm{SL}}\right),
$$

is the dielectric function of the superlattice, with the dc field $E_{\mathrm{SL}}$ being applied to the device, ${ }^{39}$ and $F_{1}\left(\omega, E_{\mathrm{SL}}\right)$ is defined by Eq. (21).

In the high-frequency limit $\epsilon_{0} / C Z_{A} \omega \rightarrow 0$ the solution of Eq. (41) coincides with the solution of the equation

$$
\epsilon\left(\omega, E_{\mathrm{SL}}\right)=0,
$$

describing the eigenfrequencies $\omega_{ \pm}^{H}$ of the hybrid plasmaBloch oscillations in a superlattice: ${ }^{\frac{1}{9}}$

$$
\begin{aligned}
& \omega_{ \pm}^{H}\left(E_{\mathrm{SL}}\right) \\
& \quad= \pm \omega_{P}\left[\frac{1}{1+\left(E_{\mathrm{SL}} / E_{P}\right)^{2}}+\left(\frac{\nu}{\omega_{P}}\right)^{2}\left(E_{\mathrm{SL}} / E_{P}\right)^{2}\right]^{1 / 2}+i \nu,
\end{aligned}
$$

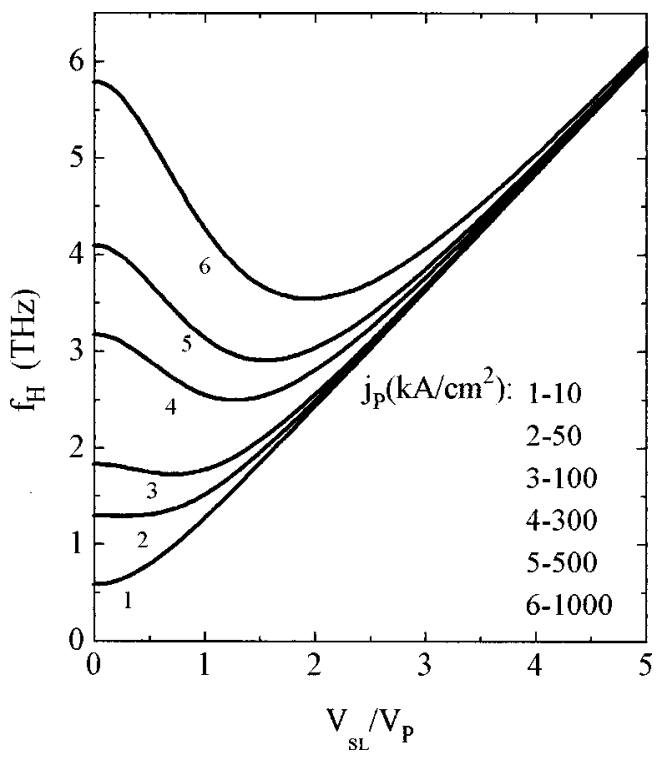

FIG. 2. The calculated hybrid plasma-Bloch oscillation frequency $f_{H}$ is plotted as a function of the normalized superlattice voltage drop $V_{\mathrm{SL}} / V_{P}$ for different values of the peak current densities $j_{P}=10,50,100,300,500$, and $1000 \mathrm{kA} / \mathrm{cm}^{2}$. Typical values of the superlattice parameters $\left(d=50 \AA, E_{P}\right.$ $=10 \mathrm{kV} / \mathrm{cm}, \epsilon_{0}=13$ ) were used for the calculations.

where $\omega_{P}$ is the plasma frequency of electrons in a superlattice. The plasma frequency $\omega_{P}$ can be given in terms of the small-field dc conductivity $\sigma_{0}$ or, equivalently, in terms of the peak current density $j_{P}$

$$
\omega_{P}=\left(\frac{4 \pi \sigma_{0} \nu}{\epsilon_{0}}\right)^{1 / 2}=\left(\frac{8 \pi j_{P} e d}{\epsilon_{0} \hbar}\right)^{1 / 2} .
$$

Equation (46) reduces in the particular case of wideminiband superlattices $\left(\Delta \gg k T, \epsilon_{F}\right)$ to the standard formula $\omega_{P}=\left(4 \pi e^{2} n / \epsilon_{0} m_{z z}\right)^{1 / 2}$.

In the limiting case of small applied dc electric fields $E_{\mathrm{SL}} / E_{P} \rightarrow 0$ one finds from Eq. (45) the plasma frequency $\omega_{ \pm}^{H} \rightarrow \pm \omega_{P}$, while in the opposite case $E_{\mathrm{SL}} / E_{P} \rightarrow \infty$, the Bloch frequency $\omega_{ \pm}^{H} \rightarrow \pm \Omega_{B}= \pm e E_{\mathrm{SL}} d / \hbar$ is recovered. The scattering frequency $\nu$ in Eq. (45) is responsible for the linewidth of the plasma-Bloch resonance.

We have calculated the hybrid plasma-Bloch oscillation frequency $f_{H}=\omega_{+}^{H} / 2 \pi$, using Eqs. (45) and (46), for the typical values of the superlattice parameters ${ }^{28-30} \epsilon_{0} \simeq 13, d$ $\simeq 50 \AA, E_{P} \simeq 10 \mathrm{kV} / \mathrm{cm}, f_{\nu}=\nu / 2 \pi=1.2 \mathrm{THz}$ for different values of the current densities $j_{p}$ (Fig. 2). For small values of the current densities $j_{P} \simeq 10 \mathrm{kA} / \mathrm{cm}^{2}$ the frequency of the hybrid oscillation increases with applied voltage in all ranges of the parameter $V_{\mathrm{SL}} / V_{P}$. On the other hand, for higher values of the current densities $j_{P} \simeq(50-1000) \mathrm{kA} / \mathrm{cm}^{2}$ the hybrid oscillation's frequency starts to decrease with increasing bias voltage in the subthreshold voltage range $V_{\mathrm{SL}} \leqslant V_{P}$. Then, at superthreshold voltages $V_{\mathrm{SL}} \geqslant V_{P}, \omega_{H}$ starts to increase again tending to the Bloch frequency. It is important to note that at high values of the dc current densities $j_{P}$ the hybrid plasma-Bloch oscillations become well defined eigenmodes of the system $\left(f_{H} \geqslant f_{\nu}\right)$. Therefore, an essential improvement of the matching efficiency between antenna and the superlat- 
tice can be expected in the high-frequency range due to a resonant excitation of this eigenmode in the device.

\section{High-frequency limit}

In the high-frequency case, when the signal frequency $f=\omega / 2 \pi$ is larger than the scattering frequency $f_{\nu}=\nu / 2 \pi$, description of the superlattice response based on the classical rectification theory is no longer valid. Let us employ Eq. (33) in order to analyze the high-frequency limit of the responsivity in the ideal case of the perfect matching and neglecting the series resistance. At $\omega \gg \nu, \Omega_{B}$, i.e., in the frequency band where ac field absorption and negative photocurrent are predominated in the superlattice response, Eq. (33) yields the frequency-independent value for the current responsivity

$$
R_{i}^{(0)}\left(\omega \rightarrow \infty, V_{\mathrm{SL}}\right)=-\frac{e}{N \hbar \nu} \frac{V_{\mathrm{SL}} / V_{P}}{1+\left(V_{\mathrm{SL}} / V_{P}\right)^{2}} .
$$

At positive bias $V_{\mathrm{SL}} / V_{P}>0$ the responsivity is negative (reduction of the dc current occurs due to dynamical localization of carriers $\left.{ }^{3-14,16}\right)$. The dc voltage dependence of the responsivity reproduces the dc Esaki-Tsu current-voltage curve. ${ }^{1}$ The responsivity reaches its maximum value $R_{i \max }^{(0)}$ $=-e /(2 N \hbar \nu)=-1 /\left(2 V_{P}\right)$ at $V_{\mathrm{SL}}=V_{P}$. For high values of the applied voltages $V_{\mathrm{SL}} \gg V_{P}$ we get $R_{i}^{(0)}\left(\omega \rightarrow \infty, V_{\mathrm{SL}} \rightarrow \infty\right)$ $=-1 / V_{\mathrm{SL}}$.

Let us compare the high-frequency limit of the responsivity of the superlattice with the quantum efficiency $R_{\max }$ $=e / \hbar \omega$ which is believed to be a fundamental restriction for the responsivity of superconductor tunnel junctions. ${ }^{19}$ This quantum efficiency (or quantum limit) corresponds to the

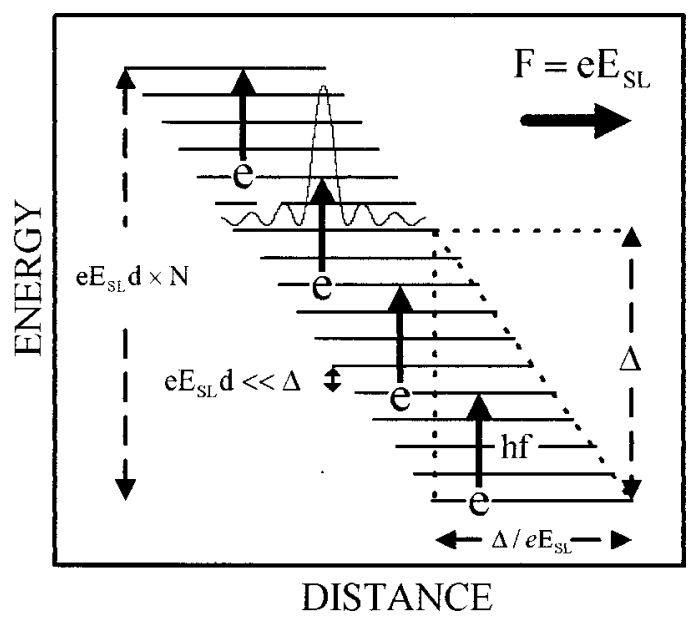

FIG. 3. Real space energy diagram illustrating THz-photon $(f \gg \nu / 2 \pi)$ detection in the superlattice: dc electric field $E_{\mathrm{SL}}$ is applied to the $n$-period semiconductor superlattice with the miniband width $\Delta$. Under the action of the dc field electrons perform Bloch oscillations with the spatial amplitude $\Delta / e E_{\mathrm{SL}}$. At critical dc electric voltage (field) $V_{\mathrm{SL}}=V_{P}=N \hbar \nu / e\left(e E_{\mathrm{SL}} d\right.$ $=\hbar \nu$ ) electrons move against the dc electric force due to absorption of photons climbing up the Wannier-Stark ladder. The energy $2 e V_{P}$ should be absorbed from external ac field in order to subtract one electron from the external circuit. One half of this energy is needed for the electron to overcome the potential barrier which is formed by the dc force, with the other half being delivered to the lattice due to energy dissipation. A quasiclassical description of the process is valid if $f \ll \Delta / \hbar$ when allowed transitions between different Wannier-Stark state exist.

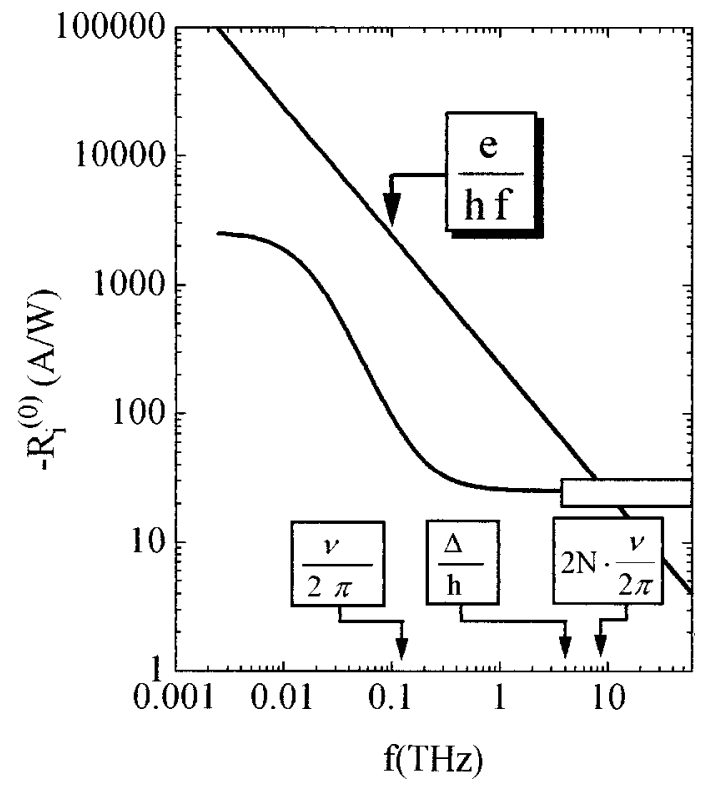

FIG. 4. The calculated current responsivity $-R_{i}^{(0)}(f)$ of the ideally coupled superlattice with $d=50 \AA, N=40, E_{P}=1 \mathrm{kV} / \mathrm{cm}, \Delta=20 \mathrm{meV}$ at subthreshold voltage $V_{\mathrm{SL}}=0.99 V_{P}$ and the quantum efficiency $e / h f$ are plotted as a function of the frequency $f=\omega / 2 \pi$. At frequency $f_{\mathrm{QL}}=2 N \nu / 2 \pi$ the value of the responsivity equals the quantum efficiency. The calculation of the responsivity is valid up to the frequency $f_{\Delta}=\Delta / h$ which should be smaller than $f_{\mathrm{QL}}=2 N \nu / 2 \pi$ within the framework of the employed model. In a wideminiband superlattice $\Delta \rightarrow 2 N \times \hbar \nu$ the responsivity can approach the quantum efficiency in the high-frequency limit.

tunneling of one electron across the junction for each signal photon absorbed, ${ }^{19}$ with a positive sign of the responsivity.

In our case the mechanism of the photon detection is different (see Fig. 3). Electrons move against the applied dc electric force due to absorption of photons. At $V_{\mathrm{SL}}=V_{P}$ the responsivity is negative, indicating that one electron is subtracted from the dc current flowing through the superlattice when the energy $2 e V_{P}$ is absorbed from the external ac field. One half of this energy is needed for the electron to overcome the potential barrier which is formed by the dc force, with another half being delivered to the lattice due to energy dissipation. If the applied dc voltage is strong enough, i.e., $V_{\mathrm{SL}} \gg V_{P}$, dissipation plays no essential role in the superlattice responsivity. In this case the energy $e V_{\mathrm{SL}}$ should be absorbed from the ac field in order to subtract one electron from the dc current simply due to the energy conservation law.

In order to demonstrate typical frequency scales involved in the problem we plot in Fig. 4 the frequency dependence of the responsivity $-R_{i}^{(0)}\left(\omega, V_{\mathrm{SL}}\right)$ for a superlattice with $d=50 \AA, N=40, L=0.2 \mu \mathrm{m}, E_{P}=1 \mathrm{kV} / \mathrm{cm}, \Delta=20$ meV, $f_{\nu}=\nu / 2 \pi=0.12 \mathrm{THz}$ at subthreshold voltage $V_{\mathrm{SL}}$ $=0.99 V_{P}$ as a function of the frequency $f=\omega / 2 \pi$. This example roughly corresponds to the superlattice samples experimentally investigated in Ref. 34. The responsivity decreases in the region $f<f_{\nu}$ and, then, tends to the constant value determined by Eq. (47). At frequency $f_{\mathrm{QL}}=2 N \nu / 2 \pi$ the value of the responsivity equals the quantum efficiency $e / h f$.

However, the semiclassical approach employed in the 
present article is restricted by the inequality $f \leqslant f_{\Delta}=\Delta / h$. This inequality simply requires that there must be an allowed transition between different Wannier-Stark states in the superlattice miniband due to photon absorption as illustrated in Fig. 3. It is important to note that $f_{\Delta} / f_{\mathrm{QL}}=\ell_{\mathrm{FP}} / L \leqslant 1$ (where $\ell_{\mathrm{FP}}=v_{0} \tau=\Delta d / 2 \hbar \nu$ is the electron's mean free path) in our calculations and, therefore, the current responsivity of the whole sample is always less than the quantum efficiency $e / h f$. In a wide-miniband superlattice with $\Delta \rightarrow 2 N \hbar \nu$ (or, equivalently, in a short superlattice sample with $L \rightarrow \ell_{\mathrm{FP}}$ ) the responsivity is approaching the quantum efficiency in the high-frequency limit.

As was mentioned above, an increase of the length $L$ of a detector may have an advantage in the reduction of the parasitic capacitance $C \propto 1 / L$, and, hence, in the increasing of the rolloff frequency $1 / R_{S} C$ of the device. It is interesting to note that in case of $N$ superconductor tunnel junctions connected in series the resulting responsivity is expected to be $e / N \hbar \omega$, implying that one photon should have been absorbed in each junction in order to add one electron to the dc circuit. ${ }^{19}$ In the case of a superlattice samples consisting of $N$ unit cells (periods) the resulting responsivity can be presented as $-e / 2 N \hbar \nu=(-e / N \hbar \omega) \times(\omega / 2 \nu)$. For $\omega>2 \nu$ the responsivity of the unit superlattice cell starts to be larger than the quantum efficiency $e / \hbar \omega$. This conclusion can be readily understood if one realizes that due to absorption of one photon in the miniband electrons can "jump" over several superlattice periods as illustrated in Fig. 3.

\section{RESULTS AND DISCUSSION}

In this section we shall investigate the current responsivity of the superlattice making use of Eq. (32) which takes into account both the finite matching efficiency between the antenna and the superlattice and the parasitic losses in the superlattice diode. We present our results using the dimensional value of $R_{i}\left(\omega, V_{\mathrm{SL}}\right)$ (given in units of $\mathrm{A} / \mathrm{W}$ ) and also introducing the normalized responsivity

$$
R_{i N}\left(\omega, V_{\mathrm{SL}}\right)=R_{i}\left(\omega, V_{\mathrm{SL}}\right) /(e / \hbar \omega),
$$

which permits us to compare directly the responsivity $R_{i}\left(\omega, V_{\mathrm{SL}}\right)$ of the superlattice with the quantum efficiency $e / \hbar \omega$. In our calculations we use the typical geometrical dimensions of the superlattice samples investigated in the experiments $^{44-46}$ and also assume that the bowtie antenna impedance $Z_{A}=50 \Omega$ does not depend on THz-photon frequency. ${ }^{34}$

\section{A. Excitation of the plasma-Bloch oscillations}

For demonstration of the frequency dependence of the superlattice current responsivity in the $\mathrm{THz}$-frequency band we will focus on the $\mathrm{GaAs} / \mathrm{Ga}_{0.5} \mathrm{Al}_{0.5} \mathrm{As}$ superlattices specially designed to operate as millimeter wave oscillators at room temperature. In Ref. 44 wide-miniband superlattice samples with $d=50 \AA, \Delta \simeq 113 \mathrm{meV}, n \simeq 10^{17} \mathrm{~cm}^{-3}$, were investigated experimentally. They demonstrated a wellpronounced Esaki-Tsu negative differential conductance for $E_{\mathrm{SL}} \geqslant E_{P} \simeq 4 \mathrm{kV} / \mathrm{cm}$ with the high peak current of the order of $j_{P} \simeq 130 \mathrm{kA} / \mathrm{cm}^{2}$. The measured value of the peak current is in good agreement with the estimate $j_{P}$

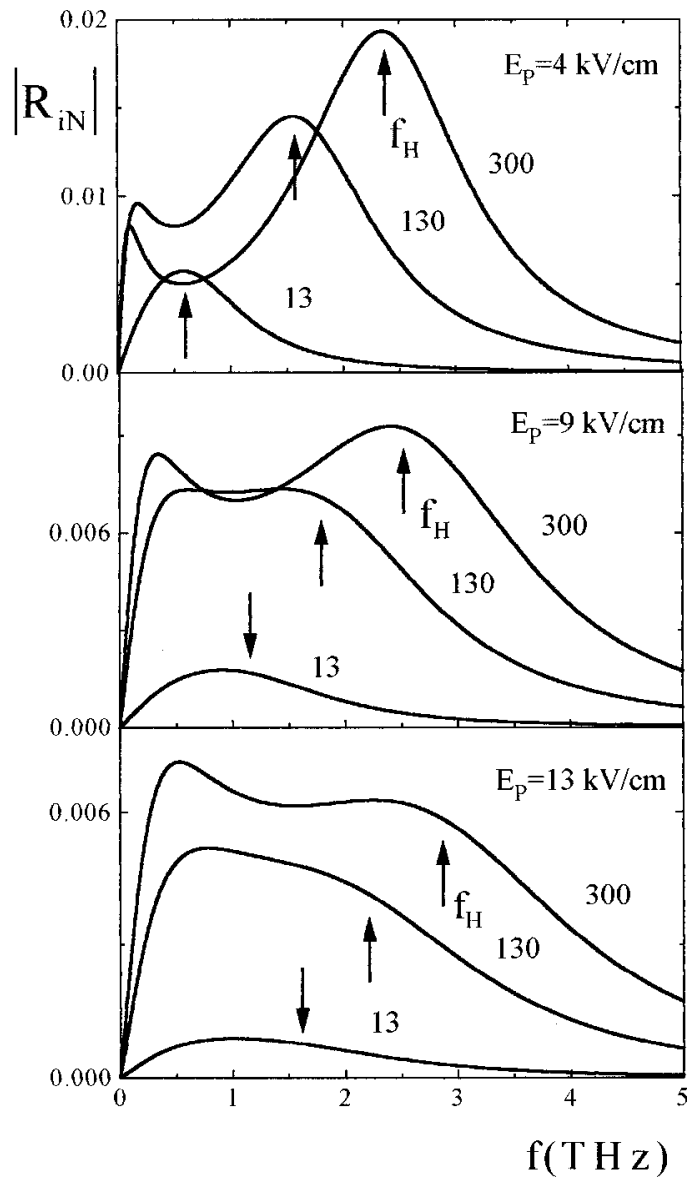

FIG. 5. The frequency dependence of the normalized current responsivity $\left|R_{i N}\right|=\left|R_{i} /(e / \hbar \omega)\right|$ of the superlattice THz-photon detector $(a=2 \mu \mathrm{m}, L$ $\left.=0.5 \mu \mathrm{m}, R_{S}=10 \Omega, V_{\mathrm{SL}}=0.95 V_{P}\right)$ is calculated for three values of the peak current density $\left(j_{P}=13,130\right.$, and $\left.300 \mathrm{kA} / \mathrm{cm}^{2}\right)$ and for three values of the peak electric field $\left(E_{P}=4,9\right.$, and $\left.13 \mathrm{kV} / \mathrm{cm}\right)$. The relevant positions of the hybrid plasma-Bloch frequencies $f_{H}$ are indicated for each curve by arrows showing characteristic resonance (high peak current densities) and rolloff (low peak current densities) behavior.

$\simeq(80-160) \mathrm{kA} / \mathrm{cm}^{2}$ for $n \simeq(1-2) \times 10^{17}, T=300 \mathrm{~K}$ based on Eq. (12), if one assumes an equilibrium Boltzmann distribution for the charge carriers. From the peak electric field and current we find the scattering and plasma frequencies $f_{\nu} \simeq 0.5 \mathrm{THz}, f_{P}=2 \mathrm{THz}$, respectively, assuming $\epsilon_{0}=13$ for the average dielectric lattice constant. The maximum frequency for the semiclassical approach to be valid for these samples is $f_{\Delta} \simeq 27 \mathrm{THz}$. Figure 5 shows the frequency dependence of the normalized current responsivity calculated for three values of the peak current density in the superlattice, i.e., $j_{P}=13,130$, and $300 \mathrm{kA} / \mathrm{cm}^{2}$ and for three values of the peak electric field, $E_{P}=4,9$, and $13 \mathrm{kV} / \mathrm{cm}$. We also use the typical values for the superlattice length $L=0.5 \mu \mathrm{m}$ (superlattice consists of 100 periods), and assume $a=2 \mu \mathrm{m}$ for the superlattice mesa radius. ${ }^{44-46}$ We choose $R_{S}=10 \Omega$ for the series resistance of the device in the THz-frequency band, i.e., the same value as for resonant tunneling diodes having the same radius of mesas. ${ }^{37}$ The calculations are performed in the region of the positive differential conductance for dc bias voltage close to the peak voltage ( $V_{\mathrm{SL}}$ $\left.=0.95 V_{P}\right)$. 


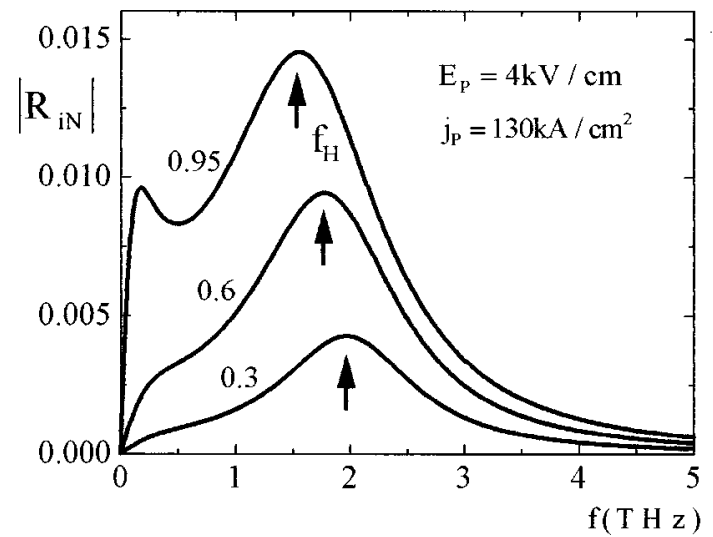

FIG. 6. The frequency dependence of the normalized current responsivity $\left|R_{i N}\right|=\left|R_{i} /(e / \hbar \omega)\right|$ of the superlattice THz-photon detector $(a=2 \mu \mathrm{m}, L$ $\left.=0.5 \mu \mathrm{m}, R_{S}=10 \Omega, E_{P}=4 \mathrm{kV} / \mathrm{cm}, J_{P}=130 \mathrm{kA} / \mathrm{cm}^{2}\right)$ is calculated for three values of the applied dc voltage $V_{\mathrm{SL}}=0.3,0.6$, and $0.95 V_{P}$. The hybrid plasma-Bloch frequencies $f_{H}$ (indicated for each dc voltage by arrows) are found to be in good agreement with positions of maximum values of the responsivity.

For $E_{P}=4 \mathrm{kV} / \mathrm{cm}\left(f_{\nu} \simeq 0.5 \mathrm{THz}\right)$ Fig. 5 demonstrates well-pronounced resonant behavior of the normalized responsivity as a function of frequency. The resonance frequency and the maximum value of the responsivity rise if the peak current density increases. For $j_{p}=300 \mathrm{kA} / \mathrm{cm}^{2}$ the normalized responsivity reaches its maximum value $-R_{i N}$ $\simeq 0.02\left(-R_{i} \simeq 2 \mathrm{~A} / \mathrm{W}\right)$ at frequency $f \simeq 2.5 \mathrm{THz}$. For higher values of the peak electric fields $E_{P}=9 \mathrm{kV} / \mathrm{cm}\left(f_{\nu}\right.$ $\simeq 1.08 \mathrm{THz})$, and $E_{P}=13 \mathrm{kV} / \mathrm{cm}\left(f_{\nu} \simeq 1.57 \mathrm{THz}\right)$ the resonance linewidths are broadened due to implicit increase of the scattering frequencies. In particular, for $E_{P}=13 \mathrm{kV} / \mathrm{cm}$, $j_{P}=300 \mathrm{kA} / \mathrm{cm}^{2}$ the normalized responsivity has an almost constant value $-R_{i N} \simeq 0.006\left(-R_{i} \simeq 0.6 \mathrm{~A} / \mathrm{W}\right)$ up to $f$ $\simeq 2.5 \mathrm{THz}$ and, then, rapidly decreases.

The frequency behavior of the normalized responsivity originates from excitation of the plasma-Bloch oscillations in the superlattice. We indicate in Fig. 5 the positions of the hybrid frequencies $f_{H}=\left|\omega_{ \pm}^{H}\right| / 2 \pi$ with arrows. For small peak electric fields (low values of the scattering frequencies) the hybrid frequency corresponds to the maximum of the normalized responsivity. For higher values of the peak field (higher values of the scattering frequencies) it corresponds to the rolloff frequency at which the responsivity starts to decline.

Frequency dependence of the responsivity for different applied dc fields, $V_{\mathrm{SL}}=0.3,0.6$, and $0.95 V_{P}$ is illustrated in Fig. 6 for $j_{P}=130 \mathrm{kA} / \mathrm{cm}^{2}$. The same geometrical dimensions and circuit parameters of the superlattice device have been chosen for calculations as previously. The normalized responsivity decreases with decreasing of the bias field tending to zero at $V_{\mathrm{SL}} \rightarrow 0$. On the other hand, the position of maximum responsivity shifts to lower values with increasing of the bias field in full agreement with Eq. (45).

\section{B. Optimized superlattice length}

The enhancement of the normalized responsivity requires an optimum matching efficiency of the superlattice to the broadband antenna and minimization of the parasitic

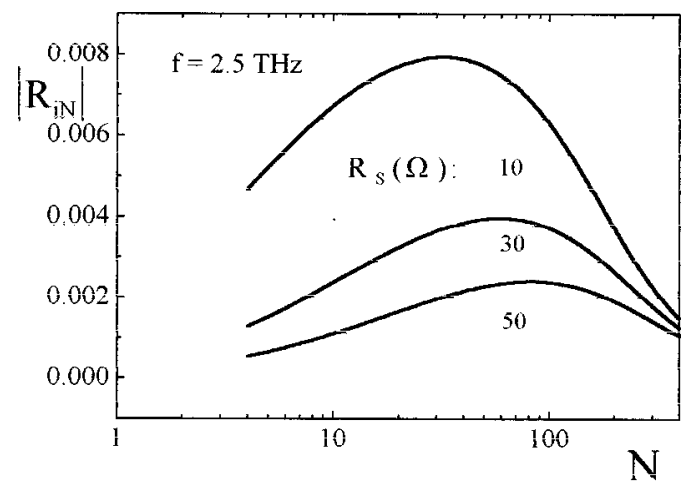

FIG. 7. The dependence of the normalized current responsivity $\left|R_{i N}\right|$ $=\left|R_{i} /(e / \hbar \omega)\right|$ of the superlattice THz-photon detector $\left(a=2 \mu \mathrm{m}, E_{P}\right.$ $\left.=4 \mathrm{kV} / \mathrm{cm}, j_{P}=130 \mathrm{kA} / \mathrm{cm}^{2}\right)$ is calculated at $f=2.5 \mathrm{THz}$ for three values of the series resistance $\left(R_{S}=10,30\right.$, and $\left.50 \Omega\right)$ as a function of number of the superlattice periods $N$

losses in the series resistor. These requirements impose an optimum length of the superlattice for each chosen frequency of the incoming $\mathrm{THz}$ photons and series resistance.

We show in Fig. 7 the dependence of the normalized responsivity on the number of the superlattice periods for $f$ $=2.5 \mathrm{THz}$. We used for calculation $a=2 \mu \mathrm{m}, j_{P}$ $=130 \mathrm{kA} / \mathrm{cm}^{2}, V_{\mathrm{SL}}=0.95 V_{P}$, and three values of the series resistance $R_{S}=10,30$, and $50 \Omega$. For all three values of the series resistance the responsivity displays a well pronounced maximum for the optimum number of the superlattice periods $N=N_{\max }$. The value of $N_{\max }$ increases with increasing of the series resistance $\left(N_{\max } \simeq 40\right.$ for $R_{S}=10 \Omega, N_{\max } \simeq 60$ for $R_{S}=30 \Omega$, and for $N_{\max } \simeq 90$ for $R_{S}=50 \Omega$ ). This result can be readily understood by recalling that a larger volume of the superlattice minimizes parasitic losses for higher values of the series resistance because of reduction of the sample's capacitance.

For incoming photon higher frequencies the parasitic losses in the superlattice device play an even more important role leading to a further increase of $N_{\max }$. Figure 8 shows the dependence of the normalized responsivity on the number of the superlattice periods for $f=3.9 \mathrm{THz}$ and the same superlattice parameters as in Fig. 7. In this case $\left(N_{\max } \simeq 70\right.$ for

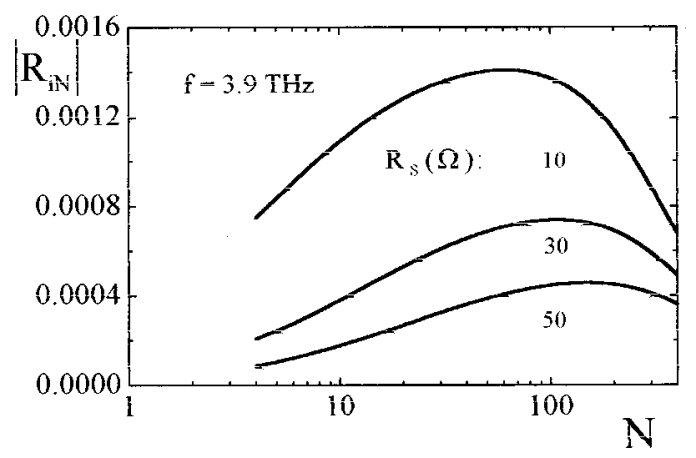

FIG. 8. The dependence of the normalized current responsivity $\left|R_{i N}\right|$ $=\left|R_{i} /(e / \hbar \omega)\right|$ of the superlattice THz-photon detector $\left(a=2 \mu \mathrm{m}, E_{P}\right.$ $\left.=4 \mathrm{kV} / \mathrm{cm}, j_{P}=130 \mathrm{kA} / \mathrm{cm}^{2}\right)$ is calculated at $f=3.9 \mathrm{THz}$ for three values of the series resistance $\left(R_{S}=10,30\right.$, and $\left.50 \Omega\right)$ as a function of number of the superlattice periods $N$. 


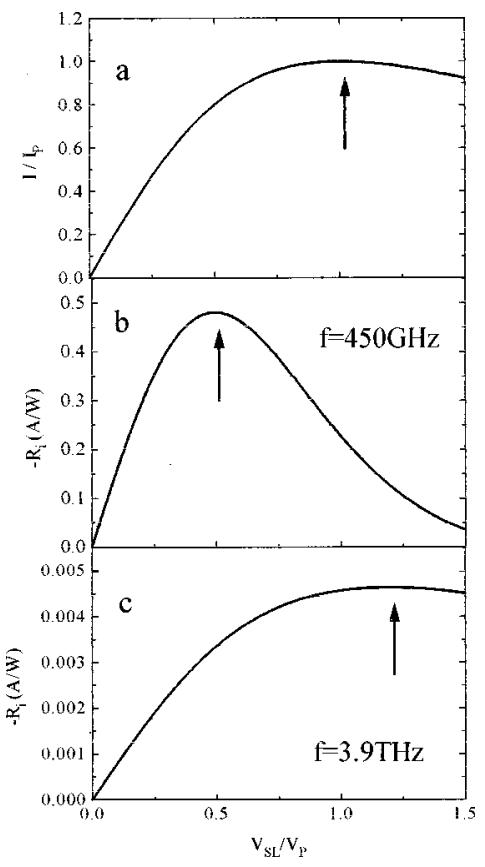

FIG. 9. (a) Normalized Esaki-Tsu $I-V$ characteristics of a superlattice THz-photon detector experimentally investigated in Ref. $41\left(a=2 \mu \mathrm{m}, E_{P}\right.$ $=13 \mathrm{kV} / \mathrm{cm}, j_{P}=10 \mathrm{kA} / \mathrm{cm}^{2}, L=0.5 \mu \mathrm{m}, R_{S}=20 \Omega$ ). Current responsivity is calculated for this detector for (b) $450 \mathrm{GHz}$ and (c) $3.9 \mathrm{THz}$ radiation as a function of the normalized dc voltage $V_{\mathrm{SL}} / V_{P}$.

$R_{S}=10 \Omega, N_{\max } \simeq 110$ for $R_{S}=30 \Omega$, and $N_{\max } \simeq 180$ for $R_{S}$ $=50 \Omega$ ). We can conclude, therefore, that the bulk mechanism of the superlattice high-frequency response provides important benefits for operation of the superlattice detectors in the THz-frequency band.

\section{Responsivity dependence on bias voltage}

Experimental studies of interaction of high-frequency fields with the miniband electrons in semiconductor superlattices having a relatively small $\left(j_{P} \simeq 10 \mathrm{kA} / \mathrm{cm}^{2}\right)$ current densities were performed ${ }^{45,46}$ for frequencies $f=90 \mathrm{GHz}, f$ $=450 \mathrm{GHz}$, and $f=3.5 \mathrm{THz}$. A field-induced reduction of the current through the superlattice was reported, with a different bias dependence below and above a characteristic frequency $(1 \mathrm{THz})$. Distinct bias dependence of the responsivity was attributed to quasistatic $(\omega \tau \ll 1)$ and dynamic $(\omega \tau$ $\gg 1$ ) interaction of the miniband electrons with ac fields at frequencies below and above $1 \mathrm{THz}$, respectively. The intraminiband relaxation time $\left(\tau \simeq 10^{-13} \mathrm{~s}\right)$ was estimated from experimental data. ${ }^{46}$

In Fig. 9 we plot the dc bias dependence of the responsivity at $f=450 \mathrm{GHz}$ and $f=3.9 \mathrm{THz}$ for the superlattice parameters corresponding to experiments ${ }^{46}\left(j_{P} \simeq 10 \mathrm{kA} / \mathrm{cm}^{2}\right.$, $E_{P} \simeq 13 \mathrm{kV} / \mathrm{cm}, \quad a \simeq 2.5 \mu \mathrm{m}, \quad L \simeq 0.5 \mu \mathrm{m}, \quad R_{S} \simeq 20 \Omega$ ) together with the dc Esaki-Tsu current-voltage curve. In a full agreement with the observations, ${ }^{46}$ at $f=450 \mathrm{GHz}$ responsivity reaches its maximum value at $V_{\mathrm{SL}} \simeq 0.5 V_{P}$ (roughly corresponding to the position of the maximum of the second derivative of the dc current-voltage curve), while for 3.9 $\mathrm{THz}$ radiation the position of the maximum is shifted to the peak voltage $V_{P}$. This can be readily understood if one takes

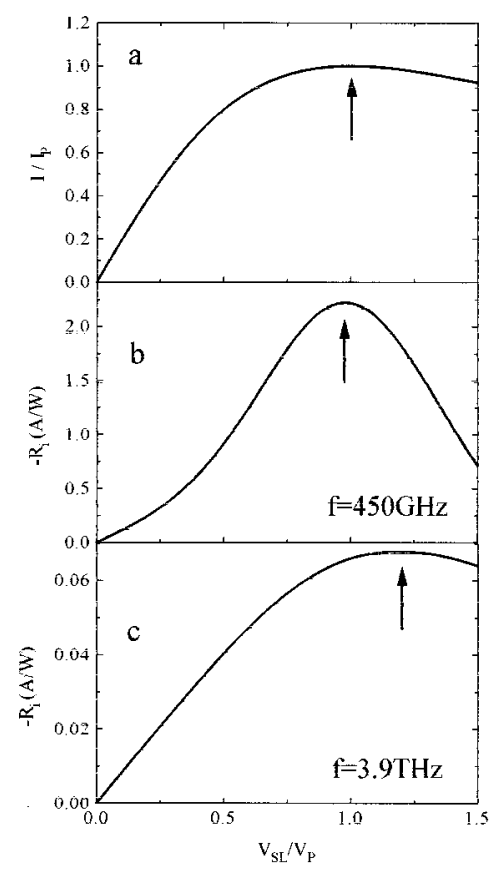

FIG. 10. (a) Normalized Esaki-Tsu $I-V$ characteristics of a high current density superlattice THz-photon detector $\left(a=2 \mu \mathrm{m}, E_{P}=4 \mathrm{kV} / \mathrm{cm}, j_{P}\right.$ $=130 \mathrm{kA} / \mathrm{cm}^{2}, L=0.5 \mu \mathrm{m}, R_{S}=20 \Omega$ ). Current responsivity is calculated for this detector for (b) $450 \mathrm{GHz}$ and (c) $3.9 \mathrm{THz}$ radiation as a function of the normalized dc voltage $V_{\mathrm{SL}} / V_{P}$.

into account that for $\omega \tau \geqslant 1$ the model of the classical rectification is no longer valid. In this case the current change under THz-photon irradiation is described by Eq. (22) giving the second derivative of the current in the finite difference form taking account of finite photon energies. At high frequencies $\omega \tau \rightarrow \infty$ this equation yields $\Delta I_{\mathrm{dc}}^{\mathrm{SL}}\left(\omega, V_{\mathrm{SL}}\right)$ $\propto I_{\mathrm{dc}}^{\mathrm{SL}}\left(V_{\mathrm{SL}}\right)$, i.e., the bias-field dependence of the responsivity should reproduce the dc current-voltage curve showing the maximum value at $V_{\mathrm{SL}} \simeq V_{P}$.

It is important to note that with increasing peak current densities this behavior qualitatively changes. Figure $10 \mathrm{dem}-$ onstrates the bias-field dependence of the responsivity for the same frequencies and superlattice parameters as Fig. 9 and for $j_{P} \simeq 130 \mathrm{kA} / \mathrm{cm}^{2}$. First, we emphasize that the responsivity is considerably higher than in the previous case both for $450 \mathrm{GHz}$ and $3.9 \mathrm{THz}$ radiation. Second, for $450 \mathrm{GHz}$ radiation the responsivity reaches its maximum value at $V_{\mathrm{SL}}$ $\simeq V_{P}$ and not at $V_{\mathrm{SL}} \simeq 0.5 V_{P}$ as previously. For the high peak current densities the superlattice impedance variation due to applied dc voltage essentially changes the coupling efficiency between antenna and superlattice. This leads to a qualitatively different behavior of the responsivity for low and high peak current densities: the latter can manifest in experiments a more efficient coupling of radiation into the superlattice.

\section{Optimized peak current density}

For a given superlattice geometry the current responsivity of the superlattice can also be enhanced by choosing an optimum value of the peak current densities. This circumstance is illustrated in Fig. 11 where we plot the responsivity 


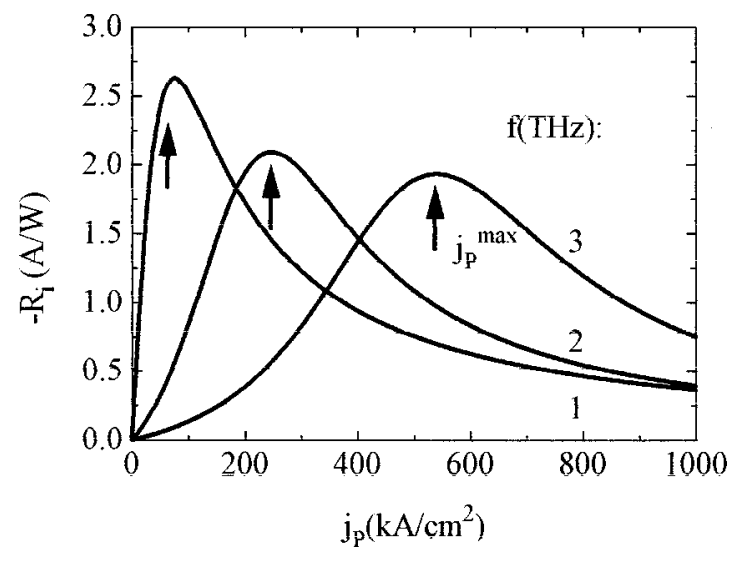

FIG. 11. The current responsivity of the superlattice $\mathrm{THz}$ photon detector $\left(a=2.5 \mu \mathrm{m}, E_{P}=4.5 \mathrm{kV} / \mathrm{cm}, L=0.5 \mu \mathrm{m}, R_{S}=20 \Omega\right)$ is calculated as a function of the peak current density for three frequencies of radiation: $f$ $=1,2$, and $3 \mathrm{THz}$. The relevant positions of the peak current densities $j_{P}^{\max }$ calculated from criterion of excitation of the plasma-Bloch oscillations $f$ $=f_{H}\left(j_{P}^{\max }\right)$ are shown for each frequency $f$, respectively.

as a function of the peak current density for $V_{\mathrm{SL}}=0.99 V_{P}$, $E_{P} \simeq 4 \mathrm{kV} / \mathrm{cm}, a \simeq 2.5 \mu \mathrm{m}, L \simeq 0.5 \mu \mathrm{m}, R_{S} \simeq 10 \Omega$ and for three frequencies of radiation, i.e., $f=1,2$, and $3 \mathrm{THz}$. For different frequencies the responsivity reaches its peaks at different values of $j_{P}^{\max }(f)$ which increases with increasing of the radiation frequency.

This behavior can also be explained by excitation of the plasma-Bloch oscillations in the superlattice by $\mathrm{THz}$ photons if one takes into account the resonant condition $f$ $=f_{H}\left(j_{P}^{\max }\right)$, where the hybrid frequency $f_{H}=\left|\omega_{ \pm}^{H} / 2 \pi\right|$ is given by Eq. (45) (see also Fig. 2). Calculated values $j_{P}^{\max }(1 \mathrm{THz}) \simeq 60 \mathrm{kA} / \mathrm{cm}^{2}, \quad j_{P}^{\max }(2 \mathrm{THz}) \simeq 220 \mathrm{kA} / \mathrm{cm}^{2}, \quad$ and $j_{P}^{\max }(3 \mathrm{THz}) \simeq 540 \mathrm{kA} / \mathrm{cm}^{2}$ are shown in Fig. 11 by arrows. They are in good agreement with the positions of the peaks found from calculations based on the general Eq. (32).

We can conclude, therefore, that high current density superlattices should be used in order to achieve the large responsivity values $(2-3 \mathrm{~A} / \mathrm{W})$ in the $1-3 \mathrm{THz}$ frequency band. The high current densities can be obtained by choosing wide miniband and/or highly doped samples. For example, according to Eq. (14) in superlattices with $\Delta \simeq 130 \mathrm{meV}^{26}$ the peak current density $j_{P} \simeq 1000 \mathrm{kA} / \mathrm{cm}^{2}$ can be reached for $n$ $\simeq 3 \times 10^{17} \mathrm{~cm}^{-3}$. In this case the equilibrium thermal excitation energy $(k T \simeq 26 \mathrm{meV})$ and the Fermi energy of degenerate electrons $\left(\epsilon_{F} \simeq 27 \mathrm{meV}\right)$ are considerably smaller than the miniband width. Hence, Eq. (14) can be employed for the peak current density estimates both for room and low temperature conditions.

Finally we would like to note that the measurements of the responsivity of resonant-tunneling (double-barrier) heterostructure diodes reported in Ref. 37 were carried out at frequencies as high as $2.5 \mathrm{THz}$. The reported value of the responsivity was of the order of several $\mu \mathrm{A} / \mathrm{W}$. As has been mentioned, ${ }^{37}$ this value is smaller by over an order of magnitude than the responsivity of THz-frequency Schottky diodes in this frequency band. We believe that an optimized superlattice detector as discussed above may have some ad- vantages over these devices due to rather high expected current responsivity in the THz-frequency band.

\section{CONCLUSIONS}

In conclusion, we have calculated the current responsivity of a semiconductor superlattice detector in the THzfrequency band based on equivalent circuit modeling. Using a path integral solution of the Boltzmann equation within the relaxation-time approximation for the collision integral we have obtained an analytical expression for the responsivity, taking account of (i) frequency dependent superlattice response to a $\mathrm{THz}$ field caused by dynamical localization of electrons, (ii) a finite matching efficiency between a detector antenna and the superlattice, and (iii) the presence of parasitic losses in the device caused by a series (contact) resistance.

We find that the responsivity of the superlattice ideally coupled to the incident radiation tends to a finite value with increasing radiation frequency. This value is simply determined by the energy conservation law governing the THzphotons absorption in the presence of scattering processes.

Excitation of plasma-Bloch oscillations in the superlattice (which are found to represent eigenmodes of the system in the THz-frequency band) can essentially enhance both the magnitude and the rolloff frequencies of the responsivity due to resonant coupling of radiation into the superlattice. The excitation of the plasma-Bloch oscillations can manifest itself as a resonance-like dependence of the normalized responsivity on the THz-photon frequency and (or) as a specific dependence of the responsivity on bias fields for superlattices showing high peak current densities.

Changes in peak current density and in superlattice length can affect drastically the coupling efficiency and parasitic losses in the superlattice device. These parameters are found to play an important role in the THz-field detector performance and need to be optimized for each value of the radiation frequency and series resistance. For higher frequencies the optimum length of the superlattice detector tends to increase demonstrating a benefit of a bulk-type mechanism of the THz-photon detection.

In currently available superlattices possessing the optimized length and the peak current the responsivity is expected to be as high as $\left|R_{i}\right| \simeq(2-3) \mathrm{A} / \mathrm{W}$ in the $1-3 \mathrm{THz}-$ frequency band. These values of the responsivity range up to several percents of the quantum efficiency $e / \hbar \omega$ of an ideal superconductor tunnel junction for this frequency range. The analysis of the current responsivity performed in this article does not necessarily assume cooling of the superlattice: the estimated values of the current responsivity of superlattices can be expected at even room temperature.

\section{ACKNOWLEDGMENTS}

The authors would like to acknowledge fruitful discussions with K. F. Renk, S. J. Allen, N. J. M. Horing, A. Wacker, E. Schomburg, J. Grenser, S. Winnerl, E. P. Dodin, 
A. Zharov, and D. G. Pavel'ev. A.A.I. gratefully acknowledges a guest professorship financed through the NATO Science Fellowship Program and the Danish Technical Research Council.

${ }^{1}$ L. Esaki and R. Tsu, IBM J. Res. Dev. 14, 61 (1970).

${ }^{2}$ R. Tsu and L. Esaki, Appl. Phys. Lett. 19, 246 (1971).

${ }^{3}$ A. A. Ignatov and Y. A. Romanov, Sov. Phys. Solid State 17, 2216 (1975); Phys. Status Solidi B 73, 327 (1976).

${ }^{4}$ V. V. Pavlovich and E. M. Epstein, Sov. Phys. Semicond. 10, 1196 (1976).

${ }^{5}$ A. A. Ignatov and Y. A. Romanov, Radiophysics and Quantum Electronics (Consultants Bureau, New York, 1978), Vol. 21, p. 90.

${ }^{6}$ D. H. Dunlap and V. M. Kenkre, Phys. Lett. A 127, 438 (1988); Phys. Rev. B 37, 6622 (1987).

${ }^{7}$ M. Holthaus, Phys. Rev. Lett. 69, 351 (1992).

${ }^{8}$ D. H. Dunlap, V. Kovanis, R. V. Duncan, and J. Simmons, Phys. Rev. B 48, 7975 (1993).

${ }^{9}$ X.-G. Zhao, X.-W. Zhang, S.-G. Chen, and W.-X. Zhang, Int. J. Mod. Phys. B 7, 4215 (1993).

${ }^{10}$ M. Wagner, Phys. Rev. B 49, 16544 (1994).

${ }^{11}$ X.-G. Zhao, R. Jahnke, and Q. Niu, Phys. Lett. A 202, 297 (1995).

${ }^{12}$ J. Rotvig, A. P. Jauho, and H. Smith, Phys. Rev. Lett. 74, 1831 (1995).

${ }^{13}$ A. A. Ignatov, E. Schomburg, J. Grenzer, K. F. Renk, and E. P. Dodin, Z. Phys. B 98, 187 (1995).

${ }^{14}$ G. Platero and R. Aguado, Appl. Phys. Lett. 70, 3564 (1997).

${ }^{15}$ R. Aguado and G. Platero, Phys. Rev. B 55, 12860 (1997).

${ }^{16}$ K. Johnsen and A. P. Jauho, Phys. Rev. B 57, 8860 (1998).

${ }^{17}$ M. Bütiker, Phys. Lett. A 96, 365 (1983).

${ }^{18}$ A. A. Ignatov, K. F. Renk, and E. P. Dodin, Phys. Rev. Lett. 70, 1996 (1993); J. B. Xia, Phys. Rev. B 58, 3565 (1998).

${ }^{19}$ For a review, see, J. R. Tucker and M. J. Feldman, Rev. Mod. Phys. 57, 1055 (1985).

${ }^{20}$ X. L. Lei, N. J. M. Horing, H. L. Cui, and K. K. Thornber, Appl. Phys. Lett. 65, 2984 (1994); Z. Phys. B 104, 221 (1997).

${ }^{21}$ A. N. Korotkov, D. V. Averin, and K. K. Liharev, Phys. Rev. B 49, 7548 (1994).

${ }^{22}$ O. A. Tkachenko, D. G. Baksheyev, and V. A. Tkachenko, J. Appl. Phys. 81, 1771 (1997).

${ }^{23}$ X. L. Lei, J. Appl. Phys. 82, 718 (1997).

${ }^{24}$ A. W. Ghosh, A. V. Kuznetsov, and J. W. Wilkins, Phys. Rev. Lett. 79, 3494 (1997)

${ }^{25}$ K. N. Alekseev, E. H. Cannon, J. C. McKinney, F. V. Kusmartsev, and D. K. Cambell, Phys. Rev. Lett. 80, 2669 (1998).
${ }^{26}$ A. Sibille, J. F. Palmier, H. Wang, and F. Mollot, Phys. Rev. Lett. 64, 52 (1990).

${ }^{27}$ H. T. Grahn, K. von Klitzing, K. Ploog, and G. H. Döhler, Phys. Rev. B 43, 12094 (1991).

${ }^{28}$ M. Hadjazi, J. F. Palmier, A. Sibille, H. Wang, E. Paris, and F. Mollot, Electron. Lett. 29, 648 (1993).

${ }^{29}$ E. Schomburg, K. Hofbeck, J. Grenser, T. Blomeier, A. A. Ignatov, K. F. Renk, D. G. Pavel'ev, Y. Koschurinov, V. Ustinov, A. Zhukov, S. Ivanov, and P. S. Kop'ev, Appl. Phys. Lett. 71, 401 (1997).

${ }^{30}$ C. Minot, N. Sahri, H. LePerson, J. F. Palmier, J. P. Medus, and J. C. Esnault, Superlattices Microstruct. 23, 1323 (1998).

${ }^{31}$ A. A. Ignatov, E. Schomburg, K. F. Renk, W. Schats, J. F. Palmier, and F. Mollot, Ann. Phys. (Leipzig) 3, 137 (1994).

${ }^{32}$ B. J. Keay, S. Zeuner, S. J. Allen, K. D. Maranowski, A. C. Gossard, U. Bhattacharaya, and M. J. W. Rodwell, Phys. Rev. Lett. 75, 4102 (1995).

${ }^{33}$ S. Zeuner, B. J. Keay, S. J. Allen, K. D. Maranowski, A. C. Gossard, U. Bhattacharaya, and M. J. W. Rodwell, Phys. Rev. B 53, 1717 (1996); A. Wacker, A. P. Jauho, S. Zeuner, and S. J. Allen, ibid. 56, 13268 (1997).

${ }^{34}$ K. Unterrainer, B. J. Keay, M. C. Wanke, S. J. Allen, D. Leopard, G. Medeiros-Ribeiro, U. Bhattacharaya, and M. J. W. Rodwell, Phys. Rev. Lett. 76, 2973 (1996); Inst. Phys. Conf. Ser. (IOP, 1997), No. 155, Chap. 10 , p. 729 .

${ }^{35}$ T. Dekorsy, R. Ott, H. Kurz, and Köhler, Phys. Rev. B 51, 17275 (1995).

${ }^{36}$ A. A. Ignatov, E. Schomburg, J. Grenzer, S. Winnerl, K. F. Renk, and E. P. Dodin, Superlattices Microstruct. 22, 15 (1997).

${ }^{37}$ T. C. L. G. Sollner, W. D. Goodhue, P. E. Tannenwald, C. D. Parker, and D. D. Peck, Appl. Phys. Lett. 43, 588 (1983).

${ }^{38}$ H. C. Torrey and C. A. Whitmer, Crystal Rectifiers (McGraw-Hill, New York, 1948), p. 336.

${ }^{39}$ A. A. Ignatov and V. I. Shashkin, Sov. Phys. JETP 66, 526 (1987).

${ }^{40}$ A. A. Ignatov and V. I. Shashkin, Phys. Status Solidi B 110, K117 (1982); Phys. Lett. A 94, 169 (1983).

${ }^{41}$ A. Wacker and A. P. Jauho, Phys. Rev. Lett. 80, 369 (1998); the conclusions on impurity scattering have recently been generalized to electronphonon scattering; A. Wacker et al. (unpublished).

${ }^{42}$ R. G. Chambers, Proc. Phys. Soc. London, Sect. A 65, 458 (1952).

${ }^{43}$ A. Wacker, S. J. Allen, J. S. Scott, M. C. Wanke, and A. P. Jauho, Phys. Status Solidi B 204, 95 (1997).

${ }^{44}$ E. Dutisseuil, A. Sibille, J. F. Palmier, F. Aristone, F. Mollot, and V. Thietty-Mieg, Phys. Rev. B 49, 5093 (1994).

${ }^{45}$ E. Schomburg, A. A. Ignatov, J. Grenser, K. F. Renk, D. G. Pavel'ev, Y. Koschurinov, B. J. Melzer, S. Ivanov, S. Schaposchnikov, and P. S. Kop'ev, Appl. Phys. Lett. 68, 1096 (1996).

${ }^{46}$ S. Winnerl et al., Phys. Rev. B 56, 10303 (1997). 